\title{
Flame Surface Fractal Characteristics in Premixed Turbulent Combustion at High Turbulence Intensities
}

\author{
Esen Cintosun* \\ University of Toronto, Toronto, Ontario M3H 5T6, Canada \\ Gregory J. Smallwoodı̀ \\ National Research Council Canada, Ottawa, Ontario K1A OR6, Canada \\ and \\ Ömer L. Gülder \\ University of Toronto, Toronto, Ontario M3H 5T6, Canada

\section{DOI: $\underline{10.2514 / 1.29533}$}

\begin{abstract}
Because of the geometrical nature of the wrinkling of the flame surfaces in premixed turbulent combustion, the fractal approach is considered to be one of the most appropriate in power-law subgrid-scale models that are widely used in large eddy simulations. However, the available experimental data in literature on fractal characteristics of the premixed turbulent flame surfaces shows significant discrepancies among data sets, and the several different fractal algorithms used for evaluation may not produce consistent results. In this work, we analyzed premixed turbulent flame front images from an extensive experimental study using three different fractal algorithms and determined the fractal parameters: namely, fractal dimension $D$, inner cutoff $\varepsilon_{i}$, and outer cutoff $\varepsilon_{o}$. These images were obtained using laser-induced fluorescence of $\mathrm{OH}$ and Mie scattering on two Bunsen-type burners with diameters of 11.2 and $22.4 \mathrm{~mm}$, with flames of propane-air with equivalence ratios of 0.8 and 1 . Nondimensional turbulence intensity $u^{\prime} / S_{L}$ (ratio of fluctuating velocity to laminar flame velocity) was from 0.9 to 15, and the Reynolds number, based on the integral length scale, was from 40 to 467. The flame front surface areas were calculated for each set of results and compared with experimental turbulent burning velocities. The three fractal algorithms used for analysis in this work gave significantly different results for the same flame image sets. Further, nondimensional turbulent burning rates computed from the fractal parameters did not agree with the experimental turbulent burning data, except for those fractal results obtained by the box-counting method and only for $u^{\prime} / S_{\mathrm{L}} \leq 6$. Implications of these results for flamelet models are discussed. In addition, the perimeter-ratio approach recently adopted to estimate the wrinkled flame surface area is discussed, and it is argued that the results obtained by the perimeter-ratio approach might not be unique.
\end{abstract}

\section{Introduction}

O $\mathrm{NE}$ of the subgrid-scale models widely used in large eddy simulation of premixed turbulent flames is the power-law approach. These are based on the flamelet models in which the reaction is assumed to take place in thin layers, separating fresh gases from burned products, being wrinkled by the turbulence [1]. In these models, the turbulent flame speed can be represented as the product of the laminar flame speed $S_{L}$, corrected for the effects of stretch (strain and curvature) and the flame-wrinkling $\Xi$. In several studies, it is proposed to model $\Xi$ using the fractal theory (see, for example [1-4]). This approach requires submodels for the fractal dimension and the inner and outer cutoffs, although the outer cutoff is dependent on the subgrid scale. Because of the geometrical nature of the wrinkling of the flame surfaces, the fractal approach is considered to be one of the most appropriate in power-law models. The flamewrinkling $\Xi$ can be expressed as

$$
\Xi=\left(A_{T} / A_{o}\right)=a\left(\varepsilon_{0} / \varepsilon_{i}\right)^{D-2}
$$

Presented as Paper 1349 at the 45th AIAA Aerospace Sciences Meeting and Exhibit, Reno, NV, 8-11 January 2007; received 29 December 2006; revision received 5 July 2007; accepted for publication 20 July 2007. Copyright @ 2007 by Ö. L. Gülder. Published by the American Institute of Aeronautics and Astronautics, Inc., with permission. Copies of this paper may be made for personal or internal use, on condition that the copier pay the $\$ 10.00$ per-copy fee to the Copyright Clearance Center, Inc., 222 Rosewood Drive, Danvers, MA 01923; include the code 0001-1452/07 \$10.00 in correspondence with the CCC.

*Graduate Student, Institute for Aerospace Studies, 4925 Dufferin Street.

Competency Leader, Combustion, Institute for Chemical Process and Environmental Technology, Building M-9, 1200 Montreal Road.

${ }^{\ddagger}$ Professor, Institute for Aerospace Studies, 4925 Dufferin Street, Member AIAA. where $\varepsilon_{0}$ and $\varepsilon_{i}$ are outer and inner cutoffs, respectively, and $D$ is the fractal dimension. The prefactor $a$ is a variable with a laminar limit of 1 and an intense turbulence limit of 2 , but may not be bounded between 1 and 2 [5]. In most modeling exercises $a$ is taken as a constant of order $1 . A_{T} / A_{o}$ is the ratio of the wrinkled flame surface area to the flow cross section.

It is argued in [6] that in the nondimensional turbulence intensity $u^{\prime} / S_{L}$ (range from 1 to 15 ) the experimentally determined mean fractal dimension is about 2.2 and does not show any dependence on turbulence intensity. This value of the fractal dimension is much lower than the values found by the previous studies that showed that the fractal dimension asymptotically reaches to $2.35-2.37$ when the nondimensional turbulence intensity exceeds 3 . It is pointed out that the probable reason for this discrepancy is the image analysis method used in the previous studies [6]. The insensitivity of the fractal dimension of flame front surfaces to turbulence intensity has been confirmed by independent experiments [묘, $\underline{8}]$. However, the conclusions reached in [6] (specifically, the insensitivity of the fractal dimension to turbulence intensity and the numerical value of the fractal dimension of the premixed turbulent flame front surfaces) have been questioned in $[\underline{8}, 9]$. To resolve the controversy regarding the magnitude of fractal dimension and its dependence, if any, on the turbulence intensity of medium to high values, further work is required. In addition, the question of whether the increase in flame surface area by turbulence is sufficient to explain the observed increases in turbulent burning rates at high enough turbulence intensities should be answered, and the conditions at which a premixed flame front is no longer a passive surface should be identified.

As discussed in [6], fractal analysis techniques based on different methods may yield different results. In this work, we analyzed previously obtained flame front images from an extensive 
experimental study using three different fractal algorithms and determined the fractal parameters: namely, fractal dimension $D$, inner cutoff $\varepsilon_{i}$, and outer cutoff $\varepsilon_{0}$. The flame front surface areas were calculated for each set of results and compared with experimental turbulent burning velocities. Additional analysis of the images consisted of determining the perimeter ratios (as implemented in $[\underline{9}, \underline{10}])$ and of comparing these ratios to turbulent burning velocities.

\section{Experimental Data}

The experimental setup used to obtain the data analyzed in this study is described in detail in [6]. A brief outline will be given here. The turbulent premixed conical flames were produced by two axisymmetric Bunsen-type burners with inner nozzle diameters of 11.2 and $22.4 \mathrm{~mm}$. Premixed turbulent propane-air flames with equivalence ratios $\Phi$ of 0.8 and 1.0 were stabilized by using an annular propane pilot for low-turbulence flames and a hydrogen pilot for high-turbulence flames. Perforated plates positioned three nozzle diameters upstream of the burner rim controlled the turbulence levels. The turbulence parameters were measured by LDV under reacting conditions in which the flow is seeded by fine silicone oil droplets. The length scales $\Lambda$ and turbulence intensities $u^{\prime}$ reported in Table 1 were measured on the burner centerline at the nozzle exit.

The instantaneous flame fronts were visualized both by laserinduced fluorescence (LIF) of $\mathrm{OH}$ and by Mie scattering. A tunable excimer laser (Lambda Physik EMG 150T MSC) was used for both techniques. The dimensions of the laser sheet at the burner centerline were about $17 \mathrm{~cm}$ by $100 \mu \mathrm{m}$ (full width at half-maximum) in the vertical and horizontal planes, respectively. The sheet thickness was less than $150 \mu \mathrm{m}$ over the full flame width. The optical detector was a large pixel format CCD detector $(1242 \times 1152$ pixels $)$ giving a flame image spatial resolution of $150 \mu \mathrm{m}$. At each condition, a minimum of 100 flame images were captured.

The flame front contours were obtained from the LIF of $\mathrm{OH}$ and Mie scattering images by the methods described in [6]. The flame front contours then were analyzed using the three different fractal algorithms described in the next section.

\section{Fractal Analysis}

In this work, we used three different implementations of the fractal analysis. These are the techniques that have been used in the majority of the applications of fractals to physical problems. The three methods are the caliper technique, Minkowski circle technique, and the box-counting technique. These are discussed in detail in [11], therefore only brief descriptions will be given here.

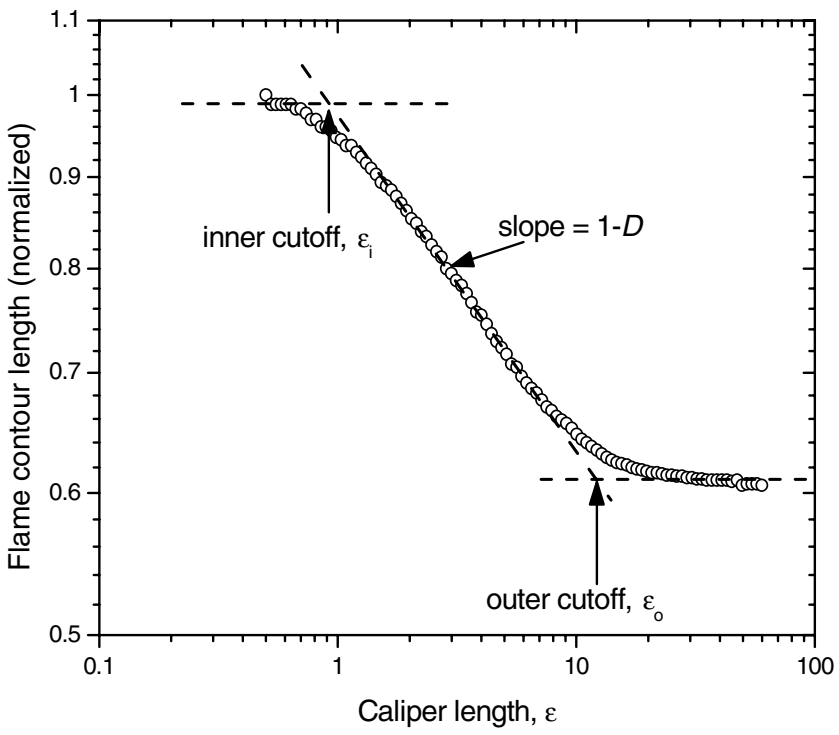

Fig. 1 Example of a Richardson plot used to evaluate the fractal parameters.

In the caliper technique, the length of a curve on a plane is estimated by counting how many ruler lengths it takes to cover the whole length of the curve from one end of the curve to the other. This process is repeated for a set of ruler lengths. A log-log plot of number of rulers (i.e., the length of the curve) versus ruler lengths yields information to determine the fractal dimension and inner and outer cutoffs. Such plots are known as Richardson plots [11]. Figure 1 shows a typical Richardson plot for one of the flame images analyzed in this study.

Fractal analysis using the Minkowski circle method involves moving a circle with a specified diameter continuously along the contour line while keeping the center of the circle on the contour line. The area covered by the circle is determined and plotted as a function of the circle diameter, for several realizations with different circle diameters, on a log-log scale to obtain the Richardson plot.

Fractal analysis using the box-counting method consists of covering the fractal image with squares (boxes) and then counting how many boxes are needed to cover the fractal curve completely. This exercise is repeated with different sizes of squares. Similar to previous two methods, a log-log plot of the number of boxes versus the box size gives the desired Richardson plot from which one can

Table 1 Summary of experimental conditions and derived fractal parameters for $\mathrm{OH}$ results

\begin{tabular}{|c|c|c|c|c|c|c|c|}
\hline Flame set- & $\Phi$ & $\Lambda, \mathrm{mm}$ & $u^{\prime} / S_{L}$ & $R e_{\Lambda}$ & Caliper method $\varepsilon_{i} / \varepsilon_{o} / D^{\underline{\mathrm{b}}}$ & Box-counting method $\varepsilon_{i} / \varepsilon_{o} / D^{\underline{b}}$ & Minkowski method $\varepsilon_{i} / \varepsilon_{o} / D^{\underline{b}}$ \\
\hline K1 & 1.0 & 1.77 & 6.47 & 308 & $0.66 / 11.39 / 2.24$ & $0.38 / 38.80 / 2.32$ & $2.82 / 19.97 / 2.39$ \\
\hline $\mathrm{K} 2$ & 1.0 & 1.77 & 6.47 & 308 & $0.62 / 10.91 / 2.23$ & $0.39 / 39.54 / 2.30$ & $2.65 / 19.68 / 2.39$ \\
\hline K3 & 0.8 & 1.91 & 9.1 & 326 & $0.45 / 9.97 / 2.19$ & $0.39 / 35.82 / 2.30$ & $3.56 / 21.04 / 2.42$ \\
\hline K4 & 1.0 & 1.65 & 10.44 & 463 & $0.59 / 11.71 / 2.24$ & $0.37 / 35.98 / 2.31$ & $1.89 / 18.70 / 2.37$ \\
\hline K5 & 0.8 & 1.66 & 15. & 467 & $0.59 / 11.16 / 2.25$ & $0.37 / 36.51 / 2.32$ & $2.12 / 19.01 / 2.40$ \\
\hline K6 & 0.8 & 1.77 & 7.73 & 257 & $0.55 / 12.67 / 2.18$ & $0.37 / 39.28 / 2.31$ & $3.30 / 20.62 / 2.38$ \\
\hline K7 & 1.0 & 1.53 & 5.3 & 218 & $0.60 / 11.44 / 2.22$ & $0.38 / 38.04 / 2.31$ & $2.68 / 20.31 / 2.38$ \\
\hline K9 & 1.0 & 1.75 & 8.58 & 404 & $0.52 / 10.44 / 2.22$ & $0.37 / 33.42 / 2.34$ & $2.31 / 20.87 / 2.38$ \\
\hline K10 & 0.8 & 1.66 & 12.9 & 402 & $0.47 / 9.38 / 2.20$ & $0.37 / 35.24 / 2.33$ & $2.71 / 20.42 / 2.40$ \\
\hline K11 & 1.0 & 1.56 & 7.09 & 297 & $0.51 / 11.03 / 2.20$ & $0.39 / 34.89 / 2.33$ & $2.63 / 20.77 / 2.39$ \\
\hline K12 & 1.0 & 1.57 & 7.86 & 332 & $0.62 / 11.32 / 2.24$ & $0.37 / 38.49 / 2.31$ & $2.84 / 21.15 / 2.38$ \\
\hline K13 & 0.8 & 1.68 & 11.27 & 355 & $0.61 / 10.03 / 2.25$ & $0.36 / 35.83 / 2.31$ & $2.29 / 21.27 / 2.38$ \\
\hline B1 & 1.0 & 1.51 & 0.84 & 34 & $1.07 / 16.98 / 2.20$ & $0.45 / 43.74 / 2.27$ & $3.87 / 28.16 / 2.33$ \\
\hline B2 & 0.8 & 1.8 & 1.5 & 50.6 & $0.58 / 23.49 / 2.12$ & $0.45 / 60.07 / 2.23$ & $7.56 / 48.89 / 2.36$ \\
\hline B3 & 0.8 & 2.48 & 2.0 & 96.1 & $1.20 / 19.42 / 2.24$ & $0.43 / 56.32 / 2.26$ & $5.01 / 40.91 / 2.37$ \\
\hline B4 & 0.8 & 1.96 & 6.16 & 227 & $0.62 / 7.77 / 2.21$ & $0.40 / 24.77 / 2.32$ & $2.55 / 16.34 / 2.38$ \\
\hline B5 & 1.0 & 2.06 & 4.31 & 239 & $0.60 / 7.61 / 2.24$ & $0.39 / 24.09 / 2.31$ & $2.21 / 15.62 / 2.37$ \\
\hline B6 & 1.0 & 1.76 & 3.27 & 155 & $0.55 / 8.96 / 2.19$ & $0.41 / 26.07 / 2.31$ & $2.75 / 17.88 / 2.38$ \\
\hline B7 & 1.0 & 1.76 & 3.27 & 155 & $0.61 / 9.35 / 2.18$ & $0.40 / 25.69 / 2.31$ & $3.09 / 18.17 / 2.38$ \\
\hline B8 & 0.8 & - & 4.55 & 145 & $0.49 / 9.29 / 2.16$ & $0.41 / 26.44 / 2.31$ & $3.16 / 19.13 / 2.37$ \\
\hline
\end{tabular}

${ }^{\mathrm{a}}$ Flame set B refers to a burner diameter of $22.4 \mathrm{~mm}$, whereas $\mathrm{K}$ refers to a burner diameter of $11.2 \mathrm{~mm}$. Only representative flames from set B are listed.

${ }^{\mathrm{b}}$ Values for $\varepsilon_{i}$ and $\varepsilon_{o}$ are in millimeters. 
evaluate the fractal parameters. The fractal dimension obtained by this method is also known as the box dimension.

The caliper technique we adopted is the algorithm used in $[\underline{7}, 8]$. The Minkowski circle technique is the implementation that was used in [12]. The box-counting technique is the implementation used in [13] and was based on the description given in [14].

\section{Results and Discussion}

One of the important aspects of planar measurements with laser sheets is that the contour information is projected on a $2 \mathrm{D}$ plane, although the flame surface is essentially three-dimensional. The laser sheet is not always perpendicular to the flame sheet, and so the front contours are projections on $2 \mathrm{D}$ planes. These contours represent a self-affine transformation of the real 3D contours because certain portions of a flame front contour are scaled by affinities, which are linear transformations with different contraction ratios in different directions. These projected contours can be classified as self-affine fractals. The statistical properties of a self-affine contour or profile $y(x)$ are invariant under the scaling transformation $x \rightarrow \xi x, y \rightarrow \xi^{H} y$ [15]. The Hurst exponent $H$ can be related to a fractal dimension of the contour through $D_{2}=2-H$ [16], whereas $D=D_{2}+1$ [11]. Thus, the 2D measurements and estimation of fractal dimension reflects the three-dimensional characteristics of the flame surfaces if we assume that, to a first approximation, the probability density function of the flame crossing angle is single modal and peaks at a value corresponding to the Hurst exponent $H$. More generally, if each contour $H$ is constant, then the contour is unifractal, where $0<H<1$. If different $H$ are needed for different segments, then the contour is multifractal [17].

Figure 2 shows the comparison of the fractal dimension $D$ evaluated for several flames images obtained by both LIF of $\mathrm{OH}$ and Mie scattering, using the box-counting method. The differences in fractal dimensions of $\mathrm{OH}$ and Mie images are within the uncertainties of $D$.

Fractal dimensions evaluated by using the caliper, box-counting, and Minkowski circle methods are shown in Figs. 3-5, respectively. In all three figures, the fractal dimension does not show any significant dependence on nondimensional turbulence intensity, especially for medium to high intensities. Another surprising observation is that the mean values of $D$ show a strong dependence on the algorithm used in extracting the fractal dimension. The caliper method yields a $D$ value close to 2.2 (Fig. 3), in agreement with the previous observations [6,7]. The fractal dimension determined by the box-counting method shows a mild dependence on $u^{\prime} / S_{L}$ for $u^{\prime} / S_{L} \leq 6$ (Fig. 4). At higher intensities, $D$ settles to a constant value

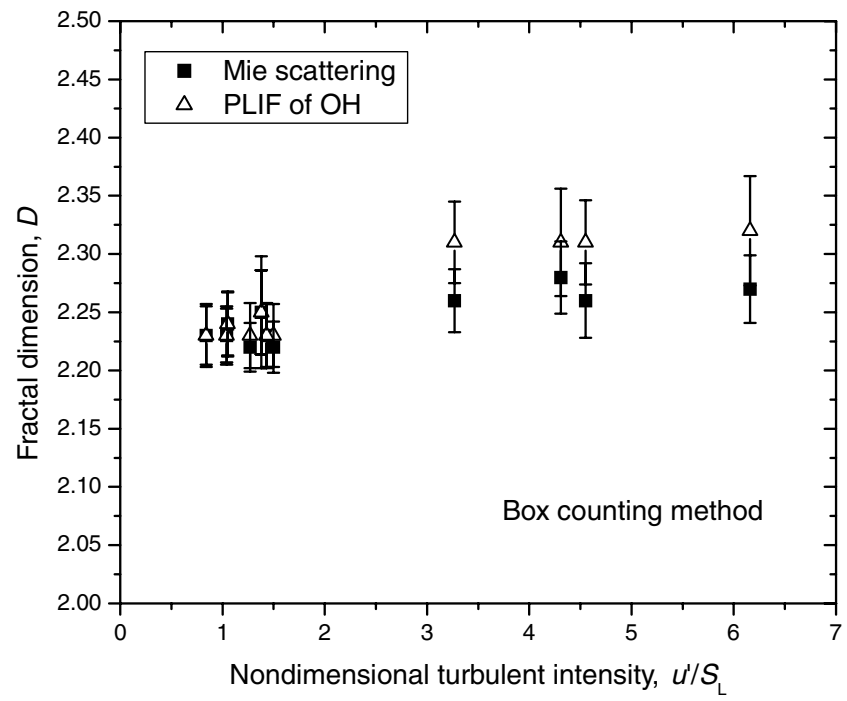

Fig. 2 Comparison of the fractal dimensions using the box-counting method from Mie scattering images and $\mathrm{OH}$ images from identical flame conditions.

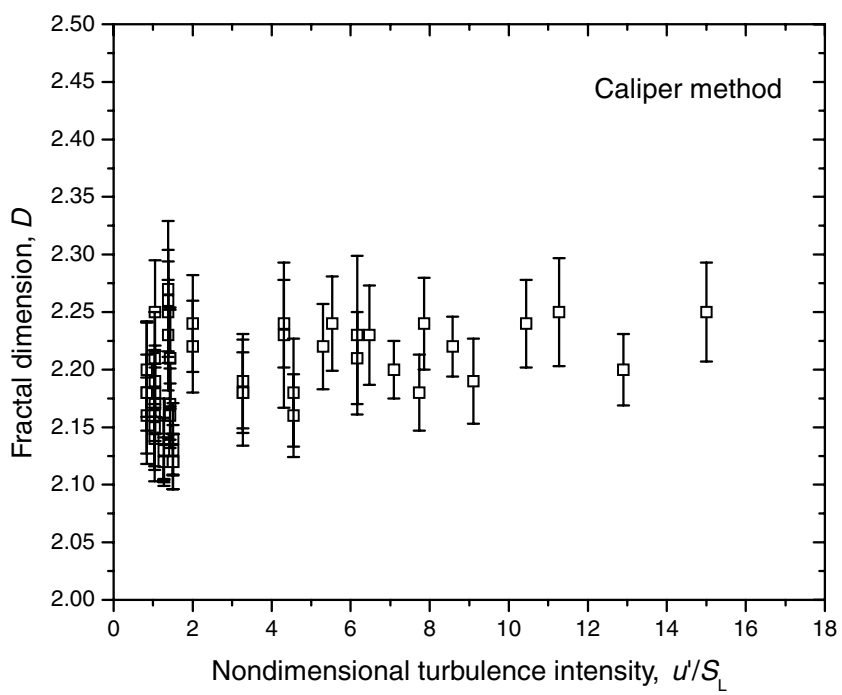

Fig. 3 Variation of the fractal dimension evaluated using the caliper method with nondimensional turbulence intensity.

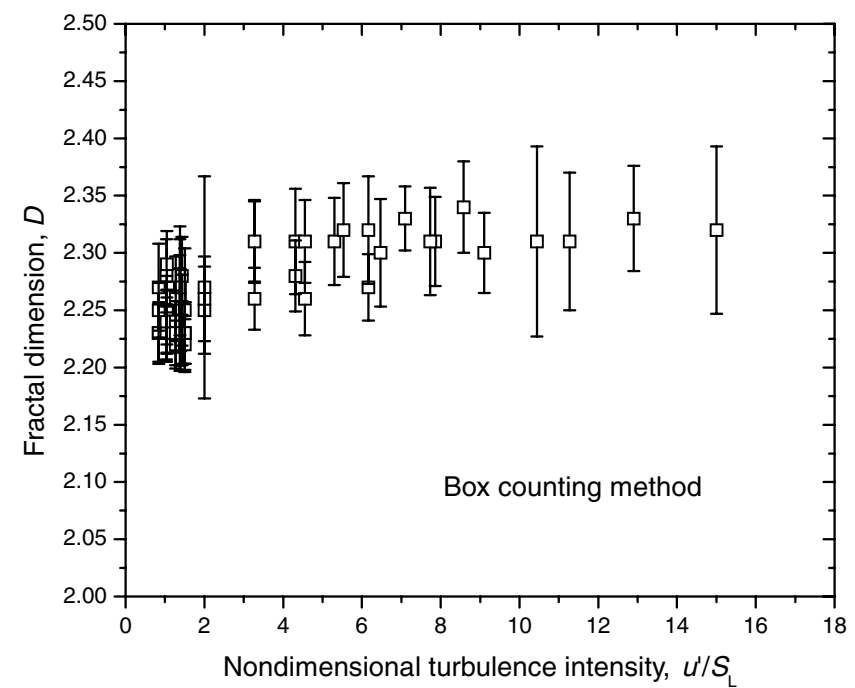

Fig. 4 Variation of the fractal dimension evaluated using the boxcounting method with nondimensional turbulence intensity.

of about 2.32. The fractal dimensions determined by the Minkowski circle method are shown in Fig. 5. Similar to box-counting results, there seems to be a very mild dependence on $u^{\prime} / S_{L}$ for $u^{\prime} / S_{L} \leq 6$, but at higher intensities, fractal dimension stays constant at about 2.4. The inner and outer cutoffs also showed variations among the estimations from three different algorithms (Table 1).

In Figs. 6-8, we compare the experimentally determined nondimensional turbulent burning velocities $S_{T} / S_{L}$ with those estimated from the measured fractal parameters, evaluated by different algorithms, using the following closure:

$$
S_{T} / S_{L}=\left(A_{T} / A_{o}\right)=a\left(\varepsilon_{0} / \varepsilon_{i}\right)^{D-2}
$$

where $a$ was taken as unity. Figure 6 compares the $S_{T} / S_{L}$ determined from the experimentally measure $\bar{d}$ values using the approach in $[6,10]$ to the nondimensional turbulent burning velocity estimated from Eq. (2) using the fractal parameters evaluated by the caliper technique. Similar comparisons are shown in Figs. 7 and 8 for $S_{T} / S_{L}$ evaluated by the box-counting and Minkowski circle methods, respectively. There is a clear and significant discrepancy between measured values of $S_{T} / S_{L}$ and those determined by Eq. (2). The only agreement seems to be for the box-counting method case (Fig. 7) for $u^{\prime} / S_{L} \leq 6$. 


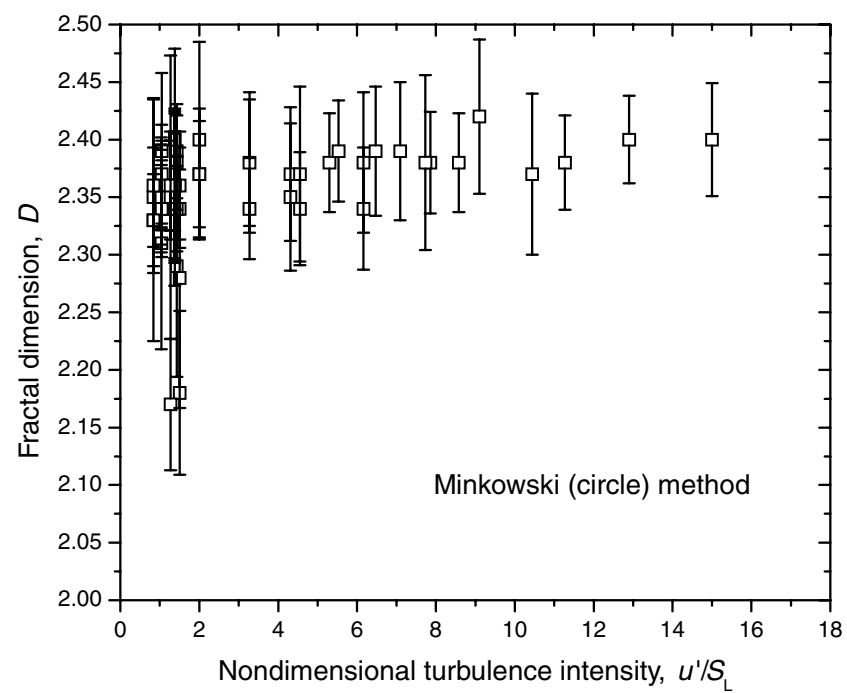

Fig. 5 Variation of the fractal dimension evaluated using the Minkowski circle method with nondimensional turbulence intensity.

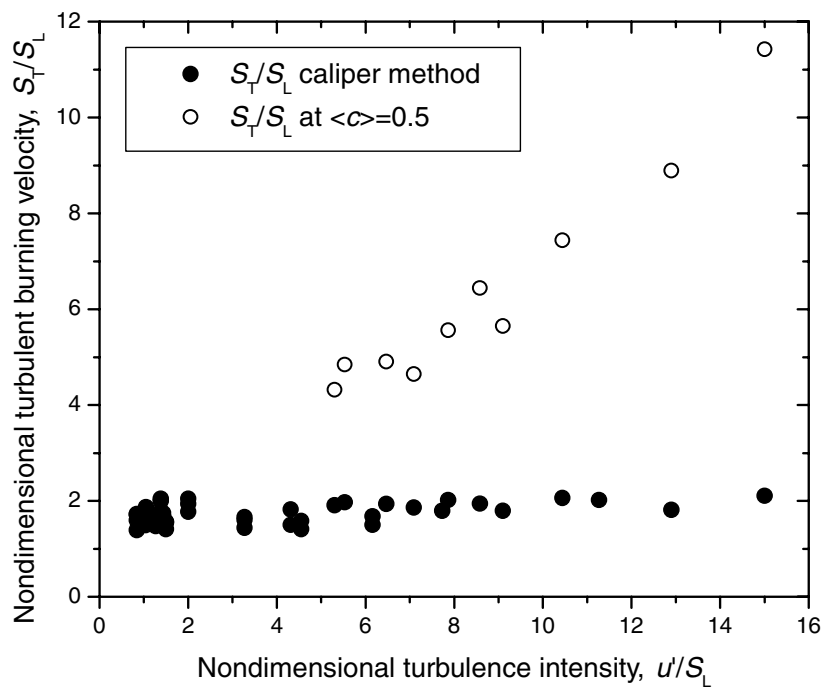

Fig. 6 The nondimensional turbulent burning velocity obtained from fractal parameters using the caliper method is compared with experimental $S_{T} / S_{L}$ data.

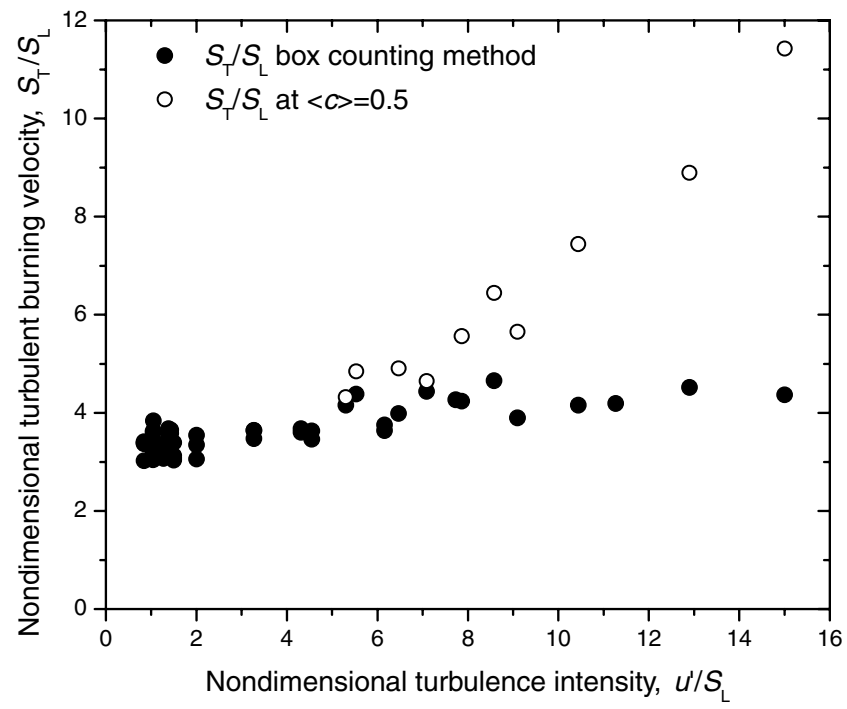

Fig. 7 The nondimensional turbulent burning velocity obtained from fractal parameters using the box-counting method is compared with experimental $S_{T} / S_{L}$ data.

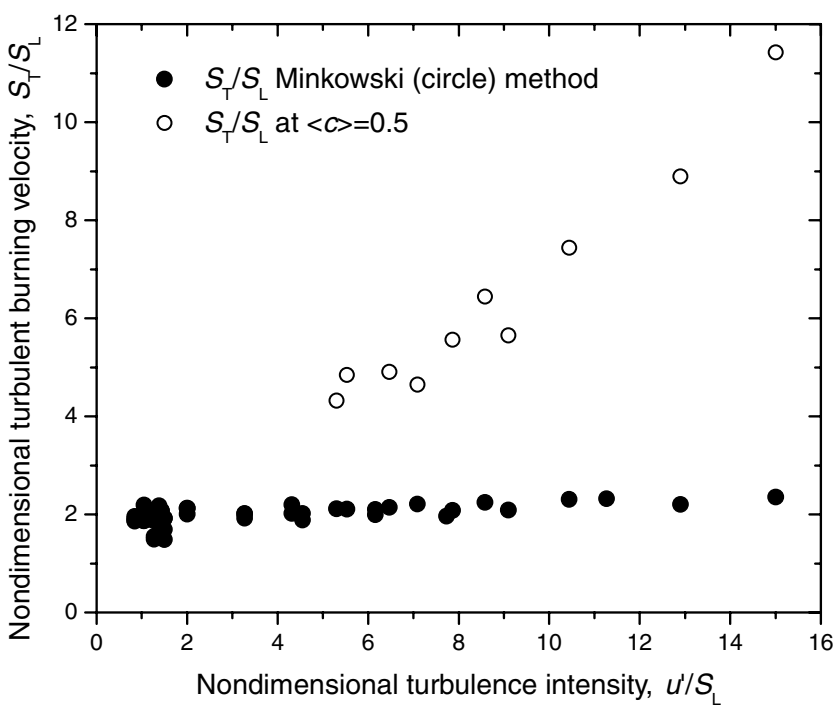

Fig. 8 The nondimensional turbulent burning velocity obtained from fractal parameters using the Minkowski circle method is compared with experimental $S_{T} / S_{L}$ data.

Several turbulent flame propagation formulations, including those based on fractal concepts, are founded on the fundamental assumption that $S_{T} / S_{L}$ is proportional to the ratio of the wrinkled flame surface area $A_{T}$ to the flow cross-sectional area $A_{o}$. If the fractal geometry approach [i.e., Eq. (1)] is yielding a true measure of the wrinkled surface area of the flame front, then Eq. (2) may not be a reasonable assumption for the turbulent premixed flames in regimes in which flamelet models are assumed to be valid. This experimental evidence can be interpreted to mean that the flame surface area increase is not the dominant mechanism in increasing the turbulent burning velocity under the conditions corresponding to the thinreaction-zones regime. When the nondimensional turbulence intensity $u^{\prime} / S_{L}$ exceeds about 6-7, the flame surface area increase estimated by the fractal analysis does not explain the observed increases in the turbulent burning velocity. Of course, this is based on the assumption that premixed turbulent flame surfaces possess fractal characteristics. In view of the fact that the ratio of outer to inner cutoff is about 10-15, fractal assumption may not be entirely justified.

A relatively new concept to get an estimate of the flame surface area is the flame perimeter ratio $P_{R}$. This approach is used in $[9,10]$. The actual length of the flame contour perimeter is divided by the flow characteristic length to estimate the surface area ratio, that is,

$$
S_{T} / S_{L}=\left(A_{T} / A_{o}\right)=b P_{R}
$$

where $b$ is a constant of unity [9]. The perimeter ratio is calculated as

$$
P_{R}=P / L_{C}
$$

where $P$ is the perimeter length of the main flame contour, and $L_{C}$ is the flow characteristic length taken as the burner diameter. This approach was also used in this work to estimate the flame surface area change with turbulence intensity. $S_{T} / S_{L}$, estimated from Eq. (3), are compared with those obtained experimentally in Fig. 9. The perimeter-ratio approach overestimates the turbulent burning velocity by more than a factor of 2 . Experimental $u^{\prime} / S_{L}$ values evaluated at mean progress variable $\langle c\rangle=0.05$ show a relatively better agreement with the perimeter-ratio estimates (Fig. 9). However, the perimeter-ratio approach is based on a central assumption that may not always be valid.

The perimeter of an irregular (non-Euclidean) planar object depends on the yardstick length used to measure it. This is the original question that led Richardson to his studies of the irregular objects: "How long is the coastline of Great Britain?" [18]. As can be seen in any Richardson plot (for example, Fig. 1), the length of the perimeter increases as the yardstick used to measure it decreases. In case of a flame front contour, the perimeter ratio measured will depend on the yardstick (caliper length, box length, or circle 


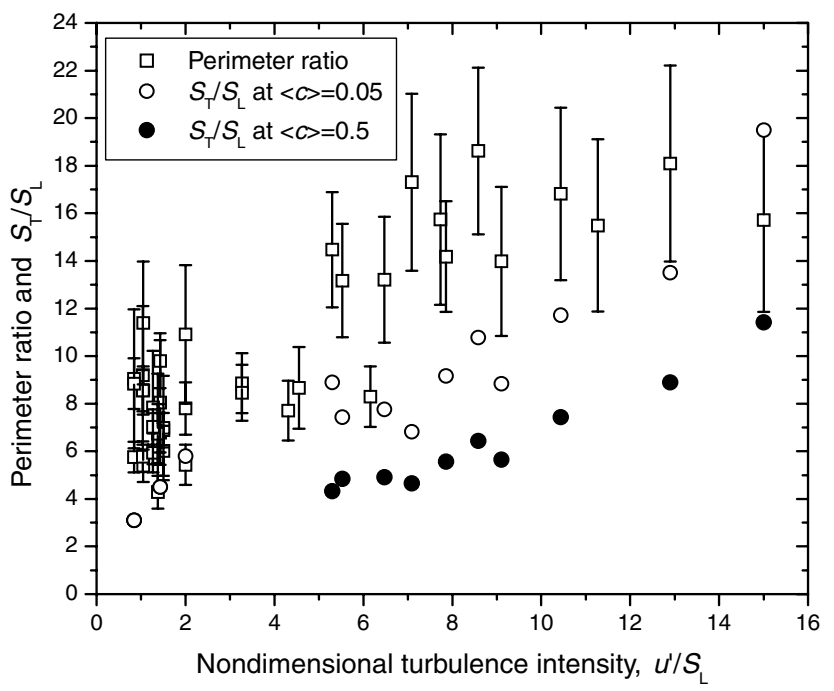

Fig. 9 The nondimensional turbulent burning velocity obtained from the perimeter ratio is compared with experimental $S_{T} / S_{L}$ data.

diameter) size and the spatial resolution of the contour image. For example, the same flame image captured by two CCD cameras with different pixel resolutions may give two different perimeter ratios. Therefore, the perimeter ratio determined as described in $[9,10]$ may not be unique and can yield incorrect flame surface area information.

\section{Conclusions}

We analyzed premixed turbulent flame front images from an extensive experimental study using three different fractal algorithms and determined the fractal parameters: namely, fractal dimension $D$, inner cutoff $\varepsilon_{i}$, and outer cutoff $\varepsilon_{0}$. The three fractal methods included the caliper technique, the box-counting method, and a third algorithm based on the Minkowski circle method. These images were obtained using laser-induced fluorescence of $\mathrm{OH}$ and Mie scattering on two Bunsen-type burners with diameters of 11.2 and $22.4 \mathrm{~mm}$, from flames of propane-air with equivalence ratios of 0.8 and 1 . Nondimensional turbulence intensity $u^{\prime} / S_{L}$ was from 0.9 to 15 , and the Reynolds number, based on the integral length scale, was from 40 to 467 . The main conclusions of this study can be summarized as follows:

1) The three fractal algorithms used for analysis in this work gave significantly different results for the same flame image set. The sensitivity of the fractal dimension to turbulence intensity was very weak for $u^{\prime} / S_{L} \geq 6$, if any, and showed a very mild dependence for $u^{\prime} / S_{L} \leq 6$

2) Nondimensional turbulent burning rates computed from the fractal parameters using the available closure did not agree with the experimental turbulent burning data. Agreement was only seen for those burning rates from fractal results obtained by the box-counting method for $u^{\prime} / S_{L} \leq 6$.

3) If the premixed turbulent flame surfaces have fractal characteristics and Eq. (1) yields a correct estimation of the flame surface area, then the current experimental results indicate that the flame surface area increase may not be the dominant mechanism in increasing the turbulent burning velocity under the conditions corresponding to the thin-reaction-zones regime. When the nondimensional turbulence intensity $u^{\prime} / S_{L}$ exceeds about $6-7$, the flame surface area increase estimated by the fractal analysis does not explain the observed increases in the turbulent burning velocity.

4) The results obtained by the perimeter-ratio approach, a method recently adopted to estimate the wrinkled flame surface area, might not be unique and could be misleading.

\section{Acknowledgments}

The work reported in this paper was supported by a Collaborative Research Opportunities (CRO) grant from the Natural Sciences and Engineering Research Council (NSERC) of Canada.

\section{References}

[1] Fureby, C., "A Fractal Flame-Wrinkling Large Eddy Simulation Model for Premixed Turbulent Combustion," Proceedings of the Combustion Institute, Vol. 30, Pt. 1, 2005, pp. 593-601. doi:10.1016/j.proci.2004.08.068

[2] Grinstein, F. F., and Fureby, C., "LES Studies of the Flow in a Swirl Gas Combustor," Proceedings of the Combustion Institute, Vol. 30, Pt. 2, 2005, pp. 1791-1798. doi:10.1016/j.proci.2004.08.082

[3] Charlette, F., Meneveau, C., and Veynante, D., "A Power-Law Flame Wrinkling Model for LES of Premixed Turbulent Combustion, Part 1: Non-Dynamic Formulation and Initial Tests," Combustion and Flame, Vol. 131, Nos. 1-2, 2002, pp. 159-180. doi:10.1016/S0010-2180(02)00400-5

[4] Charlette, F., Meneveau, C., and Veynante, D., "A Power-Law Flame Wrinkling Model for LES of Premixed Turbulent Combustion, Part 1: Dynamic Formulation," Combustion and Flame, Vol. 131, Nos. 1-2, 2002, pp. 181-197.

doi:10.1016/S0010-2180(02)00401-7

[5] Gouldin, F. C., "An Application of Fractals to Modeling Premixed Turbulent Flames," Combustion and Flame, Vol. 68, No. 3, 1987, pp. 249-266. doi:10.1016/0010-2180(87)90003-4

[6] Gülder, Ö. L., Smallwood, G. J., Wong, R., Snelling, D. R., Smith, R., Deschamps, B. M., and Sautet, J. C., "Flame Front Surface Characteristics in Turbulent Premixed Propane/Air Combustion," Combustion and Flame, Vol. 120, No. 4, 2000, pp. 407-416. doi:10.1016/S0010-2180(99)00099-1

[7] Yang, S. I., and Shy, S. S., "Measurement of Fractal Properties of Premixed Turbulent Flames and Their Relation to Turbulent Burning Velocities," Chinese Journal of Mechanics, Series A, Vol. 17, No. 2, 2001, pp. 93-101.

[8] Chen, Y.-C., and Mansour, M. S., "Geometric Interpretation of Fractal Parameters Measured in Turbulent Premixed Bunsen Flames," Experimental and Thermal Fluid Science, Vol. 27, No. 4, 2003, pp. 409-416.

[9] Lee, T. W., and Lee, S. J., "Direct Comparison of Turbulent Burning Velocity and Flame Surface Properties in Turbulent Premixed Flames," Combustion and Flame, Vol. 132, No. 3, 2003, pp. 492-502. doi:10.1016/S0010-2180(02)00495-9

[10] Filatyev, S. A., Driscoll, J. F., Carter, C. D., and Donbar, J. M., "Measured Properties of Turbulent Premixed Flames for Model Assessment, Including Burning Velocities, Stretch Rates, and Surface Densities," Combustion and Flame, Vol. 141, Nos. 1-2, 2005, pp. 121. doi:10.1016/j.combustflame.2004.07.010

[11] Mandelbrot, B. B., The Fractal Geometry of Nature, W. H. Freeman, New York, 1982, Chap. 5, pp. 28, 33 .

[12] Foucher, F., and Mounaim-Rousselle, C., "Fractal Approach to the Evaluation of Burning Rates in the Vicinity of the Piston in a SparkIgnition Engine," Combustion and Flame, Vol. 143, No. 3, 2005, pp. 323-332. doi:10.1016/j.combustflame.2005.06.007

[13] Cintosun, E., "Analysis of Premixed Turbulent Flame Front Images Obtained by Mie Scattering and Laser-Induced Fluorescence of OH," M.A.Sc. Thesis, Inst. for Aerospace Studies, Univ. of Toronto, Toronto, Ontario, Canada, Jan. 2006.

[14] Flemisch, B., "Fractal Dimension," Matlab Database [online database], http://matlabdb.mathematik.uni-stuttgart.de/index.jsp [retrieved Aug. 2007].

[15] Zaiser, M., Grasset, F. M., Koutsos, V., and Aifantis, E. C., "Self-Affine Surface Morphology of Plastically Deformed Metals," Physical Review Letters, Vol. 93, No. 19, 2004, p. 195507. doi:10.1103/PhysRevLett.93.195507

[16] Mandelbrot, B. B., "Self-Affine Fractals and Fractal Dimension," Physica Scripta, Vol. 32, No. 4, 1985, pp. 257-260. doi:10.1088/0031-8949/32/4/001

[17] Mandelbrot, B. B., and Frame, M., "Fractals," Encyclopedia of Physical Science and Technology, 3rd ed., edited by R. E. Meyers, Vol. 6, Academic Press, New York, 2001, pp. 185-207.

[18] Mandelbrot, B. B., "How Long is the Coast of Britain? Statistical SelfSimilarity and Fractional Dimension," Science, Vol. 156, No. 3775, 1967, pp. 636-638.

doi: $10.1126 /$ science. 156.3775 .636
C. Kaplan

Associate Editor 